\title{
Microsurgical treatment of arteriovenous malformations in pediatric patients: the Boston Children's Hospital experience
}

\author{
Bradley A. Gross, MD, Armide Storey, BS, Darren B. Orbach, MD, PhD, R. Michael Scott, MD, \\ and Edward R. Smith, MD
}

Department of Neurological Surgery, Boston Children's Hospital and Harvard Medical School, Boston, Massachusetts

\begin{abstract}
OBJECT Outcomes of microsurgical treatment of arteriovenous malformations (AVMs) in children are infrequently reported across large cohorts.

METHODS The authors undertook a retrospective review of departmental and hospital databases to obtain the medical data of all patients up to 18 years of age who were diagnosed with cerebral AVMs. Demographic and AVM angioarchitectural characteristics were analyzed, and for the patients who underwent surgery, the authors also analyzed the estimated intraoperative blood loss, postoperative angiographically confirmed obliteration rates, and neurological complications and outcomes classified according to the modified Rankin Scale (mRS).
\end{abstract}

RESULTS Of 117 children with cerebral AVMs, 94 underwent microsurgical resection (80\%). Twenty (21\%) of these 94 patients underwent adjunctive preoperative embolization. The overall postoperative angiographically confirmed obliteration rate was $94 \%$. As part of a new protocol, the last 50 patients in this series underwent immediate perioperative angiography, improving the subsequent obliteration rate from $86 \%$ to $100 \%(p=0.01)$. No other factors, such as a hemorrhagic AVM, size of the AVM, location, drainage, or Spetzler-Martin grade, had a statistically significant impact on the obliteration rate. Perioperative neurological deficits occurred in $17 \%$ of the patients, but the vast majority of these $(77 \%)$ were predictable visual field cuts. Arteriovenous malformations that were hemorrhagic or located in noneloquent regions were each associated with lower rates of postoperative neurological complications ( $p=0.05$ and 0.002 , respectively). In total, $94 \%$ of the children had good functional outcomes (mRS Scores $0-2$ ), and these outcomes were significantly influenced by the mRS score on presentation before surgery $(p=0.01)$. A review of 1 - and 5 -year follow-up data indicated an overall annual hemorrhage rate of $0.3 \%$ and a recurrence rate of $0.9 \%$.

CONCLUSIONS Microsurgical resection of AVMs in children is associated with high rates of angiographically confirmed obliteration and low rates of significant neurological complications. Implementation of a protocol using perioperative angiography in this series led to complete radiographically confirmed obliteration of all AVMs, with low annual repeat hemorrhage and recurrence rates.

http://thejns.org/doi/abs/10.3171/2014.9.PEDS146

KEY WORDS AVM; arteriovenous malformation; angiography; surgery; microsurgery; embolization; pediatric; vascular disorders

$\mathrm{C}$ HILDREN with arteriovenous malformations (AVMs) face a lifetime risk of life-threatening intracranial hemorrhage along with symptoms such as chronic headaches, seizures, and progressive neurological deficits due to focal cerebral steal phenomena., ${ }^{5,18,33}$ Effective counseling about treatment options and anticipated results depends on a clear understanding of the procedural morbidity and obliteration rates. Reports of treatment outcome in moderate- to large-size pediatric cohorts are rare. $2,5,16,18,22,27,31$ We reviewed our own surgical experience with AVMs to elucidate obliteration rates and treatment results in the context of an evolved institutional protocol.

\section{Methods \\ Patients and Study Design}

With the approval of the Boston Children's Hospital Institutional Review Board, we undertook a retrospective review of departmental and hospital databases to identify all patients up to 18 years of age in whom cerebral AVMs

ABBREVIATIONS AVM = arteriovenous malformation; $\mathrm{mRS}=$ modified Rankin Scale.

SUBMITTED January 4, 2014. ACCEPTED September 22, 2014.

INCLUDE WHEN CITING Published online October 31, 2014; DOI: 10.3171/2014.9.PEDS146.

DISCLOSURE The authors report no conflict of interest concerning the materials or methods used in this study or the findings specified in this paper. This study received support from the Stuart and Jane Weitzman Vascular Anomalies Center Fund. 
were diagnosed from 1995 through October 2013. For this period, complete radiographic and clinical records were available from all databases. Patients with arteriovenous fistulas, vein of Galen malformations, and spinal vascular malformations of any type were excluded from the analysis. We reviewed the records of the patients to identify the following variables: patient age at presentation, sex, mode of presentation, AVM angioarchitectural characteristics (that is, location, size, venous drainage, and associated aneurysms), embolization procedures, early and late angiographically confirmed obliteration rates, the estimated surgical blood loss, and neurological complications. The neurological conditions at presentation and at last follow-up were classified by the modified Rankin Scale $(\mathrm{mRS}){ }^{39}$ Statistical analysis for categorical variables was performed with the Fisher exact test, and comparisons between mean values of continuous variables were performed with a 2 -sided t-test.

\section{Surgical Technique}

Prior to incision, we ensured that matched blood products were in the operating room, that the microscope was draped, and that temporary aneurysm clips were selected and loaded. In all cases, a craniotomy was performed, ensuring that the margins of the lesion lay well within the margins of the operative exposure, and the dissection was performed under the operating microscope once the dura was opened. When feasible, an early identified superficial draining vein could be followed proximally to localize the nidus. Easily identifiable and major arterial feeders were then coagulated. ${ }^{24}$ An anatomical, circumferential cortical and subcortical dissection was performed with serial coagulation of supplying arterial pedicles before disconnecting the venous drainage. Indocyanine green angiography was variably employed to help distinguish arterial feeders from arterialized veins, identify en passage vessels, and to confirm resection. Since 2008, we have adopted a formal protocol in which all patients remain under general anesthesia and are transferred to the dedicated angiography suite (50 patients in this study were treated with this protocol). If a residual AVM is detected, the patient is returned to the operating room for AVM resection; otherwise, anesthesia is reversed, and extubation is performed in the angiography suite.

\section{Embolization Technique}

As part of the new protocol, since 2008, all children were evaluated for potential preoperative embolization before microsurgical resection. In most cases, embolization was performed on the day prior to microsurgical resection, with the patient being monitored in the intensive care unit overnight. This timing allowed for a full day and early start for the subsequent resection and was appropriate for most cases; some small AVMs were embolized, followed immediately by resection in the same anesthesia period. All of these procedures were performed under general anesthesia.

The goal of the embolization was to deeply penetrate the nidus with the embolic agent (most commonly Onyx [ev3]) while carefully avoiding occlusion of the venous drainage. Arterial pedicles were chosen on the basis of the access they provide to the AVM nidus and their location. Deep pedicles that were not expected to be encountered along the surgical track from the periphery to the AVM were systematically embolized if feasible. If resection was scheduled for the following day, a dexmedetomidine drip was initiated during the embolization session and then continued after a "deep" extubation. This procedure ensured strict blood pressure control while allowing for serial neurological examinations until surgery the following morning.

\section{Results \\ Overall Population and Rationale for Nonoperative Management}

Our overall cohort consisted of 117 pediatric patients with cerebral AVMs (Table 1). The cohort displayed no significant bias in the sex ratio ( $47 \%$ male), and presentation included hemorrhage in $52 \%$ of the cases, headache without hemorrhage in $20 \%$, seizures in the absence of hemorrhage in $12 \%$, and an asymptomatic/incidental finding in $16 \%$. Four of the children had hereditary hemorrhagic telangiectasia (HHT), and 2 had a family history of cerebral AVMs without a diagnosis of HHT. By mRS score, $68 \%$ of patients were functionally independent (that is, their score was 0-2). Two children died as a result of their hemorrhage, 7 were referred for stereotactic radiosurgery because the AVM was located in deep or eloquent cortex, and the remaining 14 patients underwent observation only (because of a high Spetzler-Martin grade or a preference expressed by the family) and/or underwent treatment at other institutions. The remaining 94 patients $(80 \%)$ were treated with microsurgical resection, 20 of whom (21\%) had adjunctive preoperative embolization.

\section{Surgical Population: Clinical and Radiographic Presentation}

The surgical cohort also showed no significant bias in the sex ratio ( $46 \%$ male). Mean age at the time of surgery was 10.9 years (SD 4.5 years, range 3 months to 18 years). Most of the patients presented with hemorrhage $(56 \%)$, and the remaining patients had headaches (18\%), seizures $(13 \%)$, or were asymptomatic (13\%). According to the mRS, $69 \%$ of the patients were independent (that is, had mRS scores of 0-2). The AVM location was lobar in $82 \%$ of the cases, cerebellar in $14 \%$, and deep supratentorial in $4 \%$. Most AVMs (76\%) were small (up to $3 \mathrm{~cm}$ in diameter), $21 \%$ were $3-6 \mathrm{~cm}$, and $3 \%$ were larger than $6 \mathrm{~cm}$ in diameter. Thirty-five percent of the AVMs had deep venous drainage, and $13 \%$ had an aneurysm either on an associated feeding artery or at an intranidal location. Classified by Spetzler-Martin grade, ${ }^{36} 19 \%$ of the AVMs were Grade I, $46 \%$ were Grade II, $32 \%$ were Grade III, and 3\% were Grade IV. Demographic and AVM angioarchitectural characteristics did not significantly differ between the surgical cohort and the cohort at large (Table 1).

\section{Surgical Outcomes}

The surgical outcomes are summarized in Table 2. Of 87 patients undergoing formal postoperative angiography, 82 showed obliteration of the AVM (94\%). In the most recent 50 cases, angiography was performed according to 
TABLE 1. Characteristics of all patients and of those undergoing surgery*

\begin{tabular}{|c|c|c|}
\hline Variable & All Patients (\%) & Surgical Cohort (\%) \\
\hline No. of patients & $117(100)$ & $94(100)$ \\
\hline Male sex & $55(47)$ & $43(46)$ \\
\hline \multicolumn{3}{|l|}{ Presentation } \\
\hline Incidental & $19(16)$ & $12(13)$ \\
\hline Headache (no hemorrhage) & $23(20)$ & $17(18)$ \\
\hline Seizure (no hemorrhage) & $14(12)$ & $12(13)$ \\
\hline Hemorrhage & $61(52)$ & $53(56)$ \\
\hline \multicolumn{3}{|l|}{ Presenting mRS score } \\
\hline 0 & $19(16)$ & $12(13)$ \\
\hline 1 & $49(42)$ & $44(47)$ \\
\hline 2 & $12(10)$ & $9(10)$ \\
\hline $0-2$ combined & $80(68)$ & $65(69)$ \\
\hline 3 & $12(10)$ & $9(10)$ \\
\hline 4 & $9(8)$ & $9(10)$ \\
\hline 5 & $14(12)$ & $11(12)$ \\
\hline 6 & $2(2)$ & $0(0)$ \\
\hline \multicolumn{3}{|l|}{ Location } \\
\hline Lobar & $92(79)$ & $77(82)$ \\
\hline Deep supratentorial & $11(9)$ & $4(4)$ \\
\hline Cerebellar & $14(12)$ & $13(14)$ \\
\hline \multicolumn{3}{|l|}{ AVM diameter† } \\
\hline Up to $3 \mathrm{~cm}$ & $60(70)$ & $52(76)$ \\
\hline $3-6 \mathrm{~cm}$ & $21(24)$ & $14(21)$ \\
\hline$>6 \mathrm{~cm}$ & $5(6)$ & $2(3)$ \\
\hline Deep venous drainage $\ddagger$ & $45(42)$ & $31(35)$ \\
\hline \multicolumn{3}{|l|}{ Spetzler-Martin grade $†$} \\
\hline I & $13(15)$ & $13(19)$ \\
\hline II & $36(42)$ & $31(46)$ \\
\hline III & $27(31)$ & $22(32)$ \\
\hline IV & $7(8)$ & $2(3)$ \\
\hline V & $3(3)$ & $0(0)$ \\
\hline Associated aneurysm§ & $12(13)$ & $9(13)$ \\
\hline
\end{tabular}

* All values indicate number (\%) of patients; in cases in which the number of patients in subgroups differs from the total numbers in the main groups (that is, all patients and the surgical cohort), the total number in each subgroup is indicated.

† The total numbers of patients assessed for this variable were 86 for all patients and 68 for the surgical cohort; percentages were calculated with these totals.

$\ddagger$ The total numbers of patients assessed for this variable were 108 for all patients and 88 for the surgical cohort; percentages were calculated with these totals.

$\S$ The total numbers of patients assessed for this variable were 91 for all patients and 72 for the surgical cohort; percentages were calculated with these totals.

the protocol described above, that is, immediately after wound closure. This immediate postoperative angiography clearly improved obliteration rates, which increased from $86 \%$ to $100 \%$ after initiation of this protocol $(\mathrm{p}=$ 0.01) (Table 3). Preoperative embolization, hemorrhagic presentation, larger AVM size, AVM location in eloquent regions, deep venous drainage, and Spetzler-Martin grades of III or IV did not significantly affect obliteration
TABLE 2. Overall surgical outcomes

\begin{tabular}{lc}
\hline \multicolumn{1}{c}{ Parameter } & Relative Rate (\%) \\
\hline Obliteration rate & $82 / 87(94)$ \\
\hline Preop embolization & $20 / 94(21)$ \\
\hline Periop angiography & $50 / 94(53)$ \\
\hline Neurological complications & $16 / 94(17)$ \\
\hline Total & $12 / 94(13)$ \\
\hline Visual field deficit & $3 / 94(3)$ \\
\hline Hemiparesis/hemiplegia & $3 / 94(3)$ \\
\hline New seizures & \\
\hline Neurological complication by Spetzler-Martin grade & $0 / 13(0)$ \\
\hline I & $5 / 31(16)$ \\
\hline II & $7 / 24(29)$ \\
\hline III-IV & \\
\hline Neurological outcome by mRS score & $45 / 94(48)$ \\
\hline 0 & $30 / 94(32)$ \\
\hline 1 & $88 / 94(94)$ \\
\hline $0-2$ &
\end{tabular}

rates in this study, but deep venous drainage tended to be associated with a higher rate of nonobliteration $(\mathrm{p}=0.11)$.

Overall, the mean estimated blood loss during AVM surgery was $212 \mathrm{ml}$ (median $150 \mathrm{ml}$ ). Surgery on hemorrhagic $(\mathrm{p}=0.02)$ and smaller $(\mathrm{p}=0.0001)$ AVMs was associated with lower operative blood loss, while preoperative embolization, AVM location, deep venous drainage, and Spetzler-Martin grade did not appear to significantly influence operative blood loss (Table 4).

Sixteen patients (17\%) had new and lasting neurological deficits after surgery, but in 12 of these patients (75\%), these deficits were anticipated visual field cuts, given that these 12 AVMs were located close to optic radiations, the calcarine cortex, or both. Three patients (3\%) had newonset seizures requiring antiepileptic medication for long-term seizure control, and 3 had new postoperative hemiparesis due to proximity of the AVMs to the motor/ premotor cortex in 2 cases and to the internal capsule in 1 case. Evaluating the impact of Spetzler-Martin grade on neurological complications, we noted that none of the patients with Grade I, 16\% with Grade II, and 29\% with Grade III or IV AVMs had neurological complications. Arteriovenous malformations in noneloquent regions $(\mathrm{p}=$ $0.002)$ and hemorrhagic AVMs $(p=0.05)$ were associated with lower postoperative rates of neurological complications (Table 5).

\section{Long-Term Follow-Up}

The mean clinical follow-up duration in this series was 3.6 years. In total, $54 \%$ of the patients were asymptomatic (that is, had an mRS score of 0) at the follow-up, compared with $13 \%$ before the surgery. Ninety-four percent of patients were clinically independent at the follow-up (mRS Score 0-2), an improvement from a rate of $69 \%$ prior to surgery; a higher mRS score before surgery significantly predisposed patients to a higher mRS score after surgery $(\mathrm{p}=0.01)$ (Table 6). Seventy-six patients had at least 1 year of clinical follow-up; 24 patients had at least 5 years 
TABLE 3. Factors for complete AVM obliteration rates*

\begin{tabular}{lccc}
\hline \multirow{2}{*}{ Characteristic/Risk Factor } & \multicolumn{2}{c}{ Obliteration Rate (\%) } & \\
\cline { 2 - 3 } & Yes & No & p Value \\
\hline Preop embolization & $19 / 19(100)$ & $63 / 68(93)$ & 0.58 \\
\hline Hemorrhagic presentation & $49 / 53(92)$ & $33 / 34(97)$ & 0.64 \\
\hline Larger AVM size ( $\geq 3 \mathrm{~cm})$ & $16 / 16(100)$ & $47 / 48(98)$ & 1.0 \\
\hline AVM in eloquent region & $48 / 51(94)$ & $34 / 36(94)$ & 1.0 \\
\hline Deep venous drainage & $25 / 28(89)$ & $53 / 54(98)$ & 0.11 \\
\hline Spetzler-Martin Grade III or IV & $22 / 22(100)$ & $41 / 42(98)$ & 1.0 \\
\hline $\begin{array}{c}\text { Use of early postop angiogra- } \\
\text { phy/new protocol }\end{array}$ & $50 / 50(100)$ & $32 / 37(86)$ & 0.01 \\
\hline
\end{tabular}

* The table compares the obliteration rates in the presence or absence of the

7 different factors and AVM characteristics.

of follow-up; and both of these groups had similar rates of clinical independence (that is, an mRS score $<3$ in $93 \%$ and $88 \%$ of patients, respectively).

Over a combined 337.3 patient-years of clinical follow-up, 3 postoperative hemorrhages were observed in 3 patients (equivalent to a $0.9 \%$ annualized rate). Two hemorrhages occurred from known residual postoperative AVMs and 1 from a recurrent AVM. Of note, none of these 3 patients underwent perioperative angiography. In all 3 cases, the patient was returned to the operating room and the residual or recurrent AVM was resected. Considering only the 82 initially angiographically confirmed and obliterated AVMs, 1 postoperative hemorrhage originated from a recurrent AVM, corresponding to an annualized hemorrhage rate of $0.3 \%$ over 288.3 patient-years of clinical follow-up.

Including both postoperative MRI and MR angiography in these 82 cases, a total of 212.1 patient-years of imaging follow-up (mean 2.6 years) was recorded, during which 2 radiographic recurrences of AVMs were observed (corresponding to a $0.9 \%$ annualized recurrence rate). In both cases, the recurrent AVM was resected. Of the 82 patients, 55 had repeated formal digital subtraction angiography 1-2 years after the surgery, with a total of 66.6 patient-years of formal angiographic follow-up over which 2 recurrences were found. Fourteen patients had MRI follow-up at least 5 years after surgery and no recurrences of AVMs were observed in these cases.

TABLE 4. Mean intraoperative blood loss*

\begin{tabular}{lccl}
\hline & \multicolumn{2}{c}{ Mean Estimated Blood } \\
\cline { 2 - 3 } Loss $(\mathrm{ml})$ & \\
\cline { 2 - 3 } Characteristic/Risk Factor & Yes & No & p Value \\
\hline Preop embolization & 280 & 191 & 0.16 \\
\hline Hemorrhagic presentation & 147 & 315 & 0.002 \\
\hline Larger size $(\geq 3 \mathrm{~cm})$ & 453 & 160 & 0.0001 \\
\hline AVM in eloquent region & 202 & 228 & 0.65 \\
\hline Deep venous drainage & 160 & 242 & 0.18 \\
\hline Spetzler-Martin Grade III or IV & 263 & 210 & 0.46 \\
\hline
\end{tabular}

* The mean estimated blood loss in all procedures was $212 \mathrm{ml}$ (median 150 $\mathrm{ml}$ ). The table compares the blood loss in the presence or absence of the 6 different factors and AVM characteristics.
TABLE 5. Rates of postoperative neurological complications*

\begin{tabular}{lccc}
\hline \multirow{2}{*}{\multicolumn{1}{c}{ Characteristic/Factor }} & \multicolumn{2}{c}{$\begin{array}{c}\text { Neurological } \\
\text { Complications (\%) }\end{array}$} & \\
\cline { 2 - 3 } & Yes & No & p Value \\
\hline Preop embolization & $3 / 20(15)$ & $13 / 74(18)$ & 1.0 \\
\hline Hemorrhagic presentation & $6 / 57(11)$ & $10 / 37(27)$ & 0.05 \\
\hline Larger AVM size $(\geq 3 \mathrm{~cm})$ & $5 / 16(31)$ & $7 / 52(13)$ & 0.14 \\
\hline AVM in eloquent region & $15 / 55(27)$ & $1 / 39(3)$ & 0.002 \\
\hline Deep venous drainage & $6 / 31(19)$ & $10 / 57(18)$ & 1.0 \\
\hline $\begin{array}{l}\text { Spetzler-Martin Grade III or IV } \\
\text { Use of early postop angiography/ } \\
\text { new protocol }\end{array}$ & $7 / 24(29)$ & $5 / 44(11)$ & 0.10 \\
\hline
\end{tabular}

* The table compares neurological complications in the presence or absence of the 7 different factors and AVM characteristics.

\section{Discussion}

Cerebral AVMs have been proposed to form as a result of the failure of primitive vascular channels to differentiate into arteries, interposed capillaries, or veins. Although their natural history has not been prospectively studied in pediatric cohorts, a recent retrospective analysis of 120 pediatric patients with AVMs reported an annual hemorrhage rate of $4 \% .^{5}$ Assuming that in our surgical cohort the AVMs were present since birth, the retrospective annual hemorrhage rate in this series was $5.5 \%$ (that is, 57 events during 1026 patient-years). However, because this rate was derived from a surgical cohort, this estimate was potentially biased toward an increased representation of more aggressive malformations. Nonetheless, these rates are similar to or slightly higher than those reported for adults. ${ }^{4,11,37}$

The overall mortality rate due to AVM rupture in children is as high as $21 \%$ in some series. ${ }^{18}$ Furthermore, AVMs may undergo dynamic changes in morphology and develop associated arterial and venous aneurysms and venous stenoses as the patient ages. Young women must also anticipate an elevated risk of hemorrhage during pregnancy,$^{10}$ and all children with an AVM must bear the psychological burden of possessing a lesion that may cause neurological devastation or even death at any time in their life. Thus, regardless of rupture status, in our opinion all AVMs in children should be evaluated for potential treatment.

\section{Patient Selection}

Of the AVMs in our series, $80 \%$ were managed surgically. Our "surgery-first" approach is based on the surgical advantages of immediate therapeutic cure and high obliteration rates. This approach is in contradistinction to the unknown long-term impact of cranial radiosurgery in children, both in regard to obliteration rate and other longterm complications of this treatment. Immediate therapeutic management is particularly advantageous in cases of ruptured AVMs, as such patients face a greater risk of rerupture, ${ }^{4,11,37}$ particularly in the first year. ${ }^{12}$ In children presenting with clinical and/or radiographic signs of herniation from an acute hemorrhage, we prefer to evacuate the clot without attempting a resection of the AVM. If feasible, after the administration of mannitol, we perform 
TABLE 6. Rates of poor neurological outcome for various AVM characteristics and surgical procedures*

\begin{tabular}{llcc}
\hline \multirow{2}{*}{\multicolumn{1}{c}{ Characteristic/factor }} & \multicolumn{2}{c}{$\begin{array}{c}\text { Neurological } \\
\text { Outcome (\%) }\end{array}$} \\
\cline { 2 - 3 } & \multicolumn{1}{c}{ Yes } & No & p Value \\
\hline mRS score $>$ 2 before surgery & $5 / 29(17)$ & $1 / 60(2)$ & 0.01 \\
\hline Preop embolization & $1 / 20(5)$ & $5 / 74(7)$ & 1.0 \\
\hline Hemorrhagic presentation & $5 / 57(9)$ & $1 / 37(3)$ & 0.40 \\
\hline Larger AVM size ( $\geq 3 \mathrm{~cm})$ & $1 / 16(6)$ & $2 / 52(4)$ & 0.56 \\
\hline AVM in eloquent region & $3 / 55(5)$ & $3 / 39(8)$ & 0.69 \\
\hline Deep venous drainage & $4 / 31(13)$ & $2 / 57(4)$ & 0.18 \\
\hline Spetzler-Martin Grade III or IV & $2 / 24(8)$ & $1 / 44(2)$ & 0.28 \\
\hline $\begin{array}{l}\text { Use of early postop angiography/new } \\
\text { protocol }\end{array}$ & $2 / 50(4)$ & $4 / 44(9)$ & 0.41 \\
\hline
\end{tabular}

* Poor neurological outcome was defined as an mRS score $>2$.

a preoperative $\mathrm{CT}$ angiogram en route to the operating room to help delineate a safe route to the clot without disrupting the AVM. Formal digital subtraction angiography and AVM treatment are performed after evacuation of the clot along an individualized treatment course based on the child's recovery.

It is interesting to note that in our series, microsurgical resection of hemorrhagic AVMs was associated with significantly lower postoperative neurological complication rates as well as lower intraoperative blood loss than resection of nonhemorrhagic AVMs. The lower neurological complication rate after surgery for ruptured AVMs is consistent with previous studies ${ }^{13,23}$ and has in fact been incorporated in a recently published AVM grading scale that includes an older patient age, nonhemorrhagic presentation, and diffuse nidus morphology as significant risk factors for surgical morbidity. ${ }^{23}$ Importantly, although new neurological deficits were noted in $17 \%$ of the patients at follow-up in this series, the vast majority-over three-quarters $(77 \%)$ - of these deficits were relatively minor and were anticipated visual field deficits after resection of AVMs. Unanticipated neurological complications were observed in 4 patients $(4 \%)-2$ having new weakness and seizures requiring antiepileptic medication, 1 having a new seizure disorder, and another having a new weakness. As expected, in our study, Spetzler-Martin grade ${ }^{36}$ did correlate with surgical morbidity; however, location in an eloquent brain region emerged independently as a statistically significant factor that had the greatest impact on postoperative complications, consistent with our experience with other vascular malformations. ${ }^{15}$

We do recommend radiosurgery for AVMs of appropriate size that are located in eloquent areas, such as the motor cortex, functional speech cortex, the basal ganglia, thalamus, and brainstem. It is intriguing that an extensive number of studies have reported using radiosurgery to manage AVMs in children. ${ }^{1,3,5,6,20,25,28,30,34,40}$ By comparison, reports of surgical AVM series, summarized in Table $7,2,5,8,9,16-18,21,22,27,31$ are sparse and contain fewer patients, but some describe better results after resection of AVMs in children than in adults. ${ }^{31}$ Furthermore, as most pediatric AVMs in nearly all series are hemorrhagic, ${ }^{2,8,9,16-18,22}$ a more aggressive, expedient therapy via microsurgery, as
TABLE 7. Literature reports of surgical treatment for AVMs in children

\begin{tabular}{lccc}
\hline \multicolumn{1}{c}{ Authors \& Year } & $\begin{array}{c}\text { No. of } \\
\text { Patients }\end{array}$ & $\begin{array}{c}\text { Obliteration } \\
\text { Rate (\%) }\end{array}$ & $\begin{array}{c}\text { Complication } \\
\text { Rate (\%) }\end{array}$ \\
\hline Bristol et al. 2006 & $64^{*}$ & 65 & 19 \\
\hline Darsaut et al. 2011 & NS† & 67 & 10 \\
\hline Fong \& Chan 1988 & 27 & NS & 15 \\
\hline Garza-Mercado et al. 1987 & 12 & 83 & 33 \\
\hline Hladky et al. 1994 & 52 & 90 & 19 \\
\hline Hoh et al. 2000 & 21 & 95 & 5 \\
\hline Humphreys et al. 1996 & 125 & 85 & NS \\
\hline Kiriş et al. 2005 & 20 & 89 & 10 \\
\hline Klimo et al. 2007 & 35 & 76 & 14 \\
\hline Nair et al. 2012 & 36 & 100 & 14 \\
\hline Sanchez-Mejia et al. 2006 & 32 & 97 & 6 \\
\hline Current series & 94 & 94 & 17 \\
\hline
\end{tabular}

NS $=$ not specified.

* From a series of 82 children undergoing any treatment modality.

† From a series of 120 children who underwent 104 surgical procedures; procedures included attempts at AVM obliteration and those aimed at only treating associated aneurysms.

opposed to radiosurgery, should be sought in such cases. It is interesting to note that in the most recent iteration of radiosurgical grading schemes, hemorrhage was considered an adverse factor for radiosurgical success,,$^{38}$ an assessment that differs from the observed impact of hemorrhage on the outcomes of microsurgery. ${ }^{23}$ We did not observe significant operative blood loss, a potential limitation of microsurgery relative to radiosurgery, and our obliteration rates were high. As underscored by our data and by those of previous series, immediate perioperative angiography appears crucial in achieving total AVM obliteration. ${ }^{2}$ Radiosurgical series may rely on noninvasive imaging modalities to reach conclusions about obliteration status of AVMs, $, 3,25,34$ and potentially delayed radiosurgical complications are of particular concern in pediatric patients..$^{14,19,29,32}$

Although the management of unruptured AVMs has come under considerable scrutiny as a result of the ARUBA study ("A Randomized trial of Unruptured Brain Arteriovenous malformations"), ${ }^{26}$ the observations in that study have little relevance to pediatric patients, given that children were excluded from the ARUBA study and that the length of its clinical follow-up was shorter (the mean length of follow-up was $<3$ years). Furthermore, although 76 of the 114 AVMs in the ARUBA interventional group (68\%) were of Spetzler-Martin Grades I or II, only 5 were treated with microsurgery (4\%) and 12 with microsurgery and embolization (11\%). This makes it difficult to draw any conclusions from the ARUBA study about the impact of microsurgical resection on unruptured AVMs compared with that of observation only. Nonetheless, a review of the ARUBA study is relevant, as its data reveal an annual stroke rate of approximately $4 \%$ for untreated AVMs.

The data reported here are important, as the natural history of untreated AVMs in children incorporates an annual hemorrhage rate of $4 \%-5.5 \%$. These percentages contrast sharply with our postsurgery annual hemorrhage 
rate of $0.3 \%$, representing a marked reduction in bleeding risk that is directly attributable to the surgical treatment. Consequently, the data of others, coupled with our experience reported here, support the practice of employing resection to improve the natural history and outcomes in children with intracranial AVMs.

\section{Rationale for AVM Embolization}

Although our results did not indicate greater obliteration rates and lower estimated blood loss after preoperative embolization, the absence of these improvements was likely an artifact of selection bias. We did not embolize all AVMs but rather only larger AVMs and those with deep arterial feeders. This selective embolization had the goal of reducing anticipated blood loss, of lowering the risk of perfusion pressure breakthrough, and of providing an Onyx-marked intraoperative guide to the lesion. Our surgical preference for Onyx stems not only from the roadmap it provides to feeding vessels at surgery, but also from the relative ease of coagulating and handling vessels embolized with Onyx as compared with $N$-butyl cyanoacrylate. In our cohort, 1 child had a postembolization hemorrhage (corresponding to a complication rate of $4.8 \%$ ); however, the child recovered well and had no long-term neurological sequelae. A recent study of 25 children undergoing 38 procedures for Onyx embolization of AVMs reported an overall obliteration rate of $12 \%$, with a procedural complication rate of $26.3 \%$ and no permanent neurological morbidity. ${ }^{35}$

Given the potential for long-term AVM recurrence in children, even if complete angiographically confirmed obliteration is serendipitously achieved, we would proceed to resection, wherever feasible, regardless of the postembolization angiographic appearance. Complete obliteration is not the primary goal of our embolization sessions. Our low embolization-related morbidity, coupled with our low total periprocedural morbidity and high AVM obliteration rates, highlights the potential advantages of adjunctive embolization used selectively in cases where an additional procedure provides a clear advantage.

\section{Utility of Perioperative Angiography}

In 2008, our institution developed a standardized protocol for the treatment of children with intracranial AVMs. An important component of this protocol is the inclusion of high-quality perioperative catheter angiography to confirm resection of the AVM prior to the conclusion of anesthesia. We observed no complications from the additional angiography. After the introduction of this protocol, the postoperative angiographically confirmed obliteration rates significantly improved from $86 \%$ to $100 \%$ ( $p=0.01$; Table 3). These results support the continued use of perioperative angiography. It is critical to emphasize that we use high-quality, biplane angiography, in contrast to the lower-quality portable units in the operating room. This markedly improves the resolution of the images and enhances the likelihood of identifying small residual AVMs.

\section{Long-Term Results}

Importantly, our series included both unruptured and ruptured AVMs with comparable results in both cohorts. Although AVMs that presented with hemorrhage did have lower volumes of operative blood loss and lower rates of perioperative neurological deficits (Tables 4 and 5), it is important to note that the presence or absence of a hemorrhage neither had a significant impact on the rate of surgical obliteration (Table 3) nor influenced the likelihood of long-term poor neurological outcomes (Table 6).

Considering a reported natural history of AVMs that includes a $4 \%-5.5 \%$ annual hemorrhage rate and an up to $20 \%$ mortality rate from each hemorrhage, we demonstrated that resection of AVMs confers a $0.3 \%$ annual hemorrhage rate and is associated with a $0.9 \%$ recurrence rate. Perioperative angiography significantly improved resection rates. Over a long period of follow-up (mean length 3.6 years), outcomes were excellent, with similar, durable 1 - and 5-year rates of clinical independence (93\% and $88 \%$, respectively).

\section{Conclusions}

This series of pediatric patients with AVMs who underwent microsurgery demonstrates high obliteration rates (94\% overall), acceptable rates of blood loss (mean blood loss $212 \mathrm{ml}$ ), and low rates of neurological complications, which were anticipated and manifested primarily as visual field deficits. Blood loss during surgery was lower for smaller and hemorrhagic AVMs. Surgeries on hemorrhagic AVMs and on AVMs in noneloquent locations were associated with lower rates of neurological complications. Long-term outcome, as measured by the mRS, was significantly influenced by the patient's mRS score at presentation. Rates of AVM obliteration improved as a result of a protocol modification mandating the use of perioperative angiography during the same anesthesia period. Surgical treatment markedly reduced the annual hemorrhage rate of AVMs compared with the rates reported from the natural history of untreated lesions. In aggregate, these data strongly support the use of resection in selected pediatric patients with intracranial AVMs.

\section{References}

1. Blamek S, Larysz D, Miszczyk L: Stereotactic linac radiosurgery and hypofractionated stereotactic radiotherapy for pediatric arteriovenous malformations of the brain: experiences of a single institution. Childs Nerv Syst 29:651-656, 2013

2. Bristol RE, Albuquerque FC, Spetzler RF, Rekate HL, McDougall CG, Zambramski JM: Surgical management of arteriovenous malformations in children. J Neurosurg (2 Suppl) 105:88-93, 2006

3. Cohen-Gadol AA, Pollock BE: Radiosurgery for arteriovenous malformations in children. J Neurosurg 104 (6 Suppl):388-391, 2006

4. da Costa L, Wallace MC, Ter Brugge KG, O'Kelly C, Willinsky RA, Tymianski M: The natural history and predictive features of hemorrhage from brain arteriovenous malformations. Stroke 40:100-105, 2009

5. Darsaut TE, Guzman R, Marcellus ML, Edwards MS, Tian L, Do HM, et al: Management of pediatric intracranial arteriovenous malformations: experience with multimodality therapy. Neurosurgery 69:540-556, 2011

6. Dinca EB, de Lacy P, Yianni J, Rowe J, Radatz MW, Preotiuc-Pietro D, et al: Gamma knife surgery for pediatric arteriovenous malformations: a 25 -year retrospective study. Clinical article. J Neurosurg Pediatr 10:445-450, 2012

7. Ellis MJ, Armstrong D, Vachhrajani S, Kulkarni AV, Dirks PB, Drake JM, et al: Angioarchitectural features associated 
with hemorrhagic presentation in pediatric cerebral arteriovenous malformations. J Neurointerv Surg 5:191-195, 2013

8. Fong D, Chan ST: Arteriovenous malformation in children. Childs Nerv Syst 4:199-203, 1988

9. Garza-Mercado R, Cavazos E, Tamez-Montes D: Cerebral arteriovenous malformations in children and adolescents. Surg Neurol 27:131-140, 1987

10. Gross BA, Du R: Hemorrhage from arteriovenous malformations during pregnancy. Neurosurgery 71:349-356, 2012

11. Gross BA, Du R: Natural history of cerebral arteriovenous malformations: a meta-analysis. Clinical article. J Neurosurg 118:437-443, 2013

12. Gross BA, Du R: Rate of re-bleeding of arteriovenous malformations in the first year after rupture. J Clin Neurosci 19:1087-1088, 2012

13. Gross BA, Duckworth EAM, Getch CC, Bendok BR, Batjer $\mathrm{HH}$ : Challenging traditional beliefs: microsurgery for arteriovenous malformations of the basal ganglia and thalamus. Neurosurgery 63:393-411, 2008

14. Gross BA, Ropper AE, Du R: Vascular complications of stereotactic radiosurgery for arteriovenous malformations. Clin Neurol Neurosurg 115:713-717, 2013

15. Gross BA, Smith ER, Goumnerova L, Proctor MR, Madsen JR, Scott RM: Resection of supratentorial lobar cavernous malformations in children. Clinical article. J Neurosurg Pediatr 12:367-373, 2013

16. Hladky JP, Lejeune JP, Blond S, Pruvo JP, Dhellemmes P: Cerebral arteriovenous malformations in children: report on 62 cases. Childs Nerv Syst 10:328-333, 1994

17. Hoh BL, Ogilvy CS, Butler WE, Loeffler JS, Putman CM, Chapman PH: Multimodality treatment of nongalenic arteriovenous malformations in pediatric patients. Neurosurgery 47:346-358, 2000

18. Humphreys RP, Hoffman HJ, Drake JM, Rutka JT: Choices in the 1990s for the management of pediatric cerebral arteriovenous malformations. Pediatr Neurosurg 25:277-285, 1996

19. Izawa M, Chernov M, Hayashi M, Nakaya K, Kamikawa S, Kato K, et al: Management and prognosis of cysts developed on long-term follow-up after Gamma Knife radiosurgery for intracranial arteriovenous malformations. Surg Neurol 68:400-406, 2007

20. Kano H, Kondziolka D, Flickinger JC, Yang HC, Flannery TJ, Awan NR, et al: Stereotactic radiosurgery for arteriovenous malformations, part 2: management of pediatric patients. Clinical article. J Neurosurg Pediatr 9:1-10, 2012

21. Kiris T, Sencer A, Sahinbas M, Sencer S, Imer M, Izgi N: Surgical results in pediatric Spetzler-Martin grades I-III intracranial arteriovenous malformations. Childs Nerv Syst 21:69-76, 2005

22. Klimo P Jr, Rao G, Brockmeyer D: Pediatric arteriovenous malformations: a 15-year experience with an emphasis on residual and recurrent lesions. Childs Nerv Syst 23:31-37, 2007

23. Lawton MT, Kim H, McCulloch CE, Mikhak B, Young WL: A supplementary grading scale for selecting patients with brain arteriovenous malformations for surgery. Neurosurgery 66:702-713, 2010

24. Maher CO, Scott RM: Linear vein-based arteriovenous malformations in children. Clinical article. J Neurosurg Pediatr 4:12-16, 2009

25. Maity A, Shu HK, Tan JE, Ruffer J, Sutton LN, Tochner Z, et al: Treatment of pediatric intracranial arteriovenous malformations with linear-accelerator-based stereotactic radiosurgery: the University of Pennsylvania experience. Pediatr Neurosurg 40:207-214, 2004

26. Mohr JP, Parides MK, Stapf C, Moquete E, Moy CS, Overbey JR, et al: Medical management with or without interventional therapy for unruptured brain arteriovenous malformations (ARUBA): a multicenter, non-blinded, randomised trial. Lancet 383:614-621, 2014
27. Nair AP, Kumar R, Mehrotra A, Srivastava AK, Sahu RN, Nair P: Clinical, radiological profile and outcome in pediatric Spetzler-Martin grades I-III arteriovenous malformations. Childs Nerv Syst 28:593-598, 2012

28. Pan DH, Kuo YH, Guo WY, Chung WY, Wu HM, Liu KD, et al: Gamma Knife surgery for cerebral arteriovenous malformations in children: a 13-year experience. J Neurosurg Pediatr 1:296-304, 2008

29. Pan HC, Sheehan J, Stroila M, Steiner M, Steiner L: Late cyst formation following gamma knife surgery of arteriovenous malformations. J Neurosurg 102 Suppl:124-127, 2005

30. Reyns N, Blond S, Gauvrit JY, Touzet G, Coche B, Pruvo JP, et al: Role of radiosurgery in the management of cerebral arteriovenous malformations in the pediatric age group: data from a 100-patient series. Neurosurgery 60:268-276, 2007

31. Sanchez-Mejia RO, Chennupati SK, Gupta N, Fullerton H, Young WL, Lawton MT: Superior outcomes in children compared with adult after microsurgical resection of brain arteriovenous malformations. J Neurosurg (2 Suppl) 105:82-87, 2006

32. Shin M, Kawahara N, Maruyama K, Tago M, Ueki K, Kirino $\mathrm{T}$ : Risk of hemorrhage from an arteriovenous malformation confirmed to have been obliterated on angiography after stereotactic radiosurgery. J Neurosurg 102:842-846, 2005

33. Smith ER, Butler WE, Ogilvy CS: Surgical approaches to vascular anomalies of the child's brain. Curr Opin Neurol 15:165-171, 2002

34. Smyth MD, Sneed PK, Ciricillo SF, Edwards MS, Wara WM, Larson DA, et al: Stereotactic radiosurgery for pediatric intracranial arteriovenous malformations: the University of California at San Francisco experience. J Neurosurg 97:4855,2002

35. Soltanolkotabi M, Schoeneman SE, Alden TD, Hurley MC, Ansari SA, DiPatri AJ Jr, et al: Onyx embolization of intracranial arteriovenous malformations in pediatric patients. Clinical article. J Neurosurg Pediatr 11:431-437, 2013

36. Spetzler RF, Martin NA: A proposed grading system for arteriovenous malformations. J Neurosurg 65:476-483, 1986

37. Stapf C, Mast H, Sciacca RR, Choi JH, Khaw AV, Connolly ES, et al: Predictors of hemorrhage in patients with untreated brain arteriovenous malformation. Neurology 66:1350-1355, 2006

38. Starke RM, Yen CP, Ding D, Sheehan JP: A practical grading scale for predicting outcome after radiosurgery for arteriovenous malformations: analysis of 1012 treated patients. Clinical article. J Neurosurg 119:981-987, 2013

39. van Swieten JC, Koudstaal PJ, Visser MC, Schouten HJ, van Gijn J: Interobserver agreement for the assessment of handicap in stroke patients. Stroke 19:604-607, 1988

40. Yeon JY, Shin HJ, Kim JS, Hong SC, Lee JI: Clinico-radiological outcomes following gamma knife radiosurgery for pediatric arteriovenous malformations. Childs Nerv Syst 27:1109-1119, 2011

\section{Author Contributions}

Conception and design: Gross, Scott, Smith. Acquisition of data: Gross, Storey. Analysis and interpretation of data: all authors. Drafting the article: Gross. Critically revising the article: Gross, Orbach, Scott, Smith. Reviewed submitted version of manuscript: all authors. Approved the final version of the manuscript on behalf of all authors: Gross. Statistical analysis: Gross. Administrative/technical/material support: Scott, Smith. Study supervision: Scott, Smith.

\section{Correspondence}

Bradley A. Gross, Department of Neurological Surgery, Boston Children's Hospital and Harvard Medical School, 300 Longwood Ave., Boston, MA 02115. email: bgross1 @ partners.org. 